\title{
Proinflammatory Stimuli Enhance Phagocytosis of Apoptotic Cells by Neutrophil Granulocytes
}

\author{
Lars Hellberg, Sabrina Fuchs, Christoph Gericke, Arup Sarkar, Martina Behnen, \\ Werner Solbach, and Tamás Laskay
}

Institute for Medical Microbiology and Hygiene, University of Lübeck, Ratzeburger Allee 160, 23538 Lübeck, Germany

Received 29 August 2011; Accepted 15 September 2011

Academic Editor: Marco Antonio Cassatella

Recently, we have reported that, in addition to macrophages, also neutrophil granulocytes can phagocytose apoptotic neutrophils. Based on this finding, we hypothesized that "cannibalistic" neutrophils at sites of acute infection/inflammation play a major role in the clearance of apoptotic neutrophils. Since at sites of infection/inflammation neutrophils are exposed to microbial constituents and proinflammatory cytokines, in the present study we analyzed the effect of TLRligands and cytokines on the ability of neutrophils to phagocytose apoptotic cells in vitro. We observed that exposure to ligands of TLR2 (Malp2, Pam3CSK4), TLR4 (LPS), TLR7/TLR8 (R848), and TLR9 (ODN 2006) led to increased phagocytosis of apoptotic cells by neutrophils. In addition, proinflammatory cytokines such as TNF and GM-CSF strongly enhanced the uptake of apoptotic cells by neutrophils. These results support the hypothesis that neutrophils acquire the ability to phagocytose apoptotic cells at sites of acute infection/inflammation and thereby can contribute to the resolution of inflammation.

KEYWORDS: Neutrophil (polymorphonuclear granulocyte), phagocytosis, apoptotic cells 


\section{INTRODUCTION}

Neutrophil granulocytes are recruited rapidly to sites of acute infection/inflammation, where they undergo apoptosis in large numbers. Since uncontrolled release of toxic substances from dead neutrophils can propagate the inflammatory response leading to tissue destruction, recognition and clearance of dying inflammatory neutrophils has a critical function for the resolution of the inflammatory response $[1,2]$. It does not only allow for the removal of the dying inflammatory cells themselves but also leads to the generation of anti-inflammatory mediators that shut down the ongoing inflammation [3,4]. Until recently, macrophages were regarded as major phagocyte population responsible for the clearance of senescent/apoptotic neutrophils [1]. However, macrophages are rare at sites of acute inflammation where the number of neutrophils within the tissue can be extremely high. Recently, we and others have reported that, in addition to macrophages, also "cannibalistic" neutrophil granulocytes can phagocytose apoptotic neutrophils [5-7].

Under physiological conditions, circulating "naïve" neutrophils have a very limited capacity to phagocytose apoptotic cells [6]. Exposed to activating stimuli such as GM-CSF [6], LPS or LPS in combination with IFN- $\gamma[5,7]$, however, neutrophils acquire the capacity to phagocytose apoptotic cells. At sites of acute infection/inflammation, neutrophils are exposed to microbial constituents and various cytokines. We hypothesize that neutrophils activated by these and other stimuli acquire an enhanced phagocytic capacity and, consequently, can play a major role in the clearance of apoptotic neutrophils. In the present study, we investigated which microbial constituents and cytokines are able to enhance the ability of neutrophils to phagocytose apoptotic cells in vitro. Isolated human neutrophils were exposed to TLR-ligands and various cytokines and their ability to phagocytose apoptotic cells was assessed in vitro. In addition, the phagocytosis of apoptotic neutrophils by neutrophils was measured in whole blood as well. We observed that exposure to ligands of TLR2 (Malp2, Pam3CSK4), TLR4 (LPS), TLR7/TLR8 (R848), and TLR9 (ODN 2006) led to an enhanced phagocytosis of apoptotic cells by neutrophils. In addition, proinflammatory cytokines such as TNF and GM-CSF strongly enhanced the uptake of apoptotic cells by neutrophils.

\section{METHODS}

\subsection{Isolation of Primary Human Neutrophils}

Peripheral blood was collected by venipuncture from healthy adult volunteers using lithium-heparin. Neutrophils were isolated as described previously [5]. The blood collection was conducted with the understanding and the consent of each participant and was approved by the ethical committee of the Medical Faculty of the University of Lübeck (05-124). The cell preparations contained $>99.9 \%$ granulocytes as determined by morphological examination of Giemsa-stained cytocentrifuge (Shandon, Pittsburgh, PA) slides. All experiments were conducted using complete medium (RPMI 1640 medium (Sigma-Aldrich, Steinheim, Germany) supplemented with $50 \mu \mathrm{M}$ 2-mercaptoethanol, $2 \mathrm{mM}$ L-glutamine, $10 \mathrm{mM}$ HEPES (all from Biochrom, Berlin, Germany); and 30\% autologous human serum). Viable neutrophils were stained with PKH67 fluorescent dye (Sigma Aldrich) as described [5].

\subsection{Induction of Neutrophil Apoptosis}

Apoptosis was induced by irradiating neutrophils $\left(2 \times 10^{7} / \mathrm{mL}\right.$ in complete medium containing $10 \%$ heat inactivated FCS instead of human serum) with $1000 \mathrm{~mJ}$ UV light (Stratalinker, Stratagene, La Jolla, CA) and subsequent incubation at $37^{\circ} \mathrm{C}$ for $4 \mathrm{~h}$. Cells obtained by this procedure were $>70 \%$ apoptotic and less than 5\% necrotic as analyzed by Annexin V-FLUOS (Roche, Mannheim, Germany)/propidium iodide (Sigma Aldrich) staining. Apoptotic neutrophils were stained with PKH26 fluorescent dye (Sigma Aldrich) for neutrophil experiments as described previously [5] and with PKH67 (Sigma Aldrich) for the whole blood experiments. 


\subsection{Phagocytosis Assays}

\subsubsection{Assessment of Ingestion of Apoptotic Neutrophils by Purified Neutrophil Granulocytes}

Freshly isolated viable neutrophils $\left(1 \times 10^{6} / \mathrm{mL}\right)$ were preincubated for $30 \mathrm{~min}$ at $37^{\circ} \mathrm{C}$ in complete medium alone or in the presence of one of the following substances: LPS $(100 \mathrm{ng} / \mathrm{mL}$, Sigma), Flagellin $(2 \mu \mathrm{g} / \mathrm{mL})$, R848 $(1 \mu \mathrm{g} / \mathrm{mL}$, both Enzo Life Sciences, Lorrach, Germany), MALP-2 (20 ng/mL), PAM3CSK4 $(40 \mu \mathrm{g} / \mathrm{mL})$, Poly I:C $(100 \mu \mathrm{g} / \mathrm{mL})$, ODN2006 or respective control $(5 \mu \mathrm{M}$, all Invivogen, San Diego, CA), IL-2 (200 ng/mL), GM-CSF (40 ng/mL), M-CSF (40 ng/mL), TNF (100 ng/mL, Peprotech, Hamburg, Germany), IL-1 $\beta$, IL-6, IL-10, IL-12, IL-17 (all 200 ng/mL, Immunotools, Friesoythe, Germany) or IL8 (200 ng/mL, R\&D Systems, Wiesbaden, Germany). Subsequently, PKH26-labeled autologous apoptotic neutrophils were added to a final concentration of $4 \times 10^{6} / \mathrm{mL}$ followed by incubation for $90 \mathrm{~min}$ at $37^{\circ} \mathrm{C}$. The tubes were placed on ice, and the phagocytosis was assessed by flow cytometry as described [5].

\subsubsection{Assessment of Ingestion of Apoptotic Neutrophils by Neutrophil Granulocytes in Whole Blood}

$50 \mu \mathrm{L}$ samples of heparinized whole blood were preincubated with the TLR-ligands and cytokines listed above for $30 \mathrm{~min}$ at $37^{\circ} \mathrm{C}$. Subsequently, $1 \times 10^{6} \mathrm{PKH} 67$-labeled apoptotic autologous neutrophils were added and the blood samples were incubated at $37^{\circ} \mathrm{C}$. After $60 \mathrm{~min}$, erythrocytes were lysed by using the BD FACS Lysing Solution (BD Biosciences). Phagocytosis of apoptotic cells by neutrophils was analyzed by flow cytometry.

\subsection{Statistical Analysis}

Statistical analysis was performed with graph pad prism 4 software. Data were analyzed using the one-way ANOVA test and Bonferroni post-test.

\section{RESULTS AND DISCUSSION}

\subsection{Exposure to Several TLR-Ligands Enhances the Phagocytosis of Apoptotic Cells by Neutrophils}

In previous studies exposure to LPS led to enhanced phagocytosis of apoptotic cells by neutrophil granulocytes $[5,7]$. To investigate whether other microbial constituents can exert similar effects, freshly isolated human neutrophils were pretreated for $30 \mathrm{~min}$ with one of the following TLR ligands: Pam3CSK4 (TLR 1/2), Malp-2 (TLR 2/6), poly I : C (TLR 3), LPS (TLR 4), flagellin (TLR 5), R848 (TLR 7,8), and the CpG DNA ODN2006 (TLR 9). Subsequently, PKH26-labeled apoptotic human neutrophils were added in a $1: 4$ ratio, and the ingestion of apoptotic neutrophils was assessed after $90 \mathrm{~min}$. In the absence of any stimulus, the rate of phagocytosis was about 20\% (Figure 1). Exposure to the TLR2 ligands Pam3CSK4 and Malp-2 as well as to the TLR4 ligand LPS led to enhanced phagocytosis of apoptotic cells (Figure 1(a)). These findings are in line with previous reports showing that ligands of TLR2 and TLR4 activate neutrophil functions including phagocytosis [8,9]. Treatment with the TLR5 ligand flagellin, however, did not lead to significant increase of the phagocytic capacity (Figure 1(a)). This may be explained with the previous observation that flagellin has a limited activating potential on neutrophil functions [8] although flagellin was shown to enhance the phagocytosis of opsonized latex beads [8].

Nucleic acids are ligands of the intracellular Toll-like receptors TLR3, TLR7, -8, and -9. In our experiments, polyinosine-polycytidylic acid (poly I : C), a synthetic analog of dsRNA, was used as TLR3 ligand, the imidazoquinoline R848 (resiquimod) was used as a potent synthetic agonist of TLR7/TLR8, and ODN2006 synthetic oligodeoxynucleotides containing unmethylated deoxycytosine-deoxyguanosine $(\mathrm{CpG})$ DNA motifs were used as ligand of TLR9. Exposure to R848 strongly enhanced the phagocytosis of apoptotic cells by neutrophils (Figure 1(a)). Similarly, CpG DNA ODN 2006 had an activating effect as well 


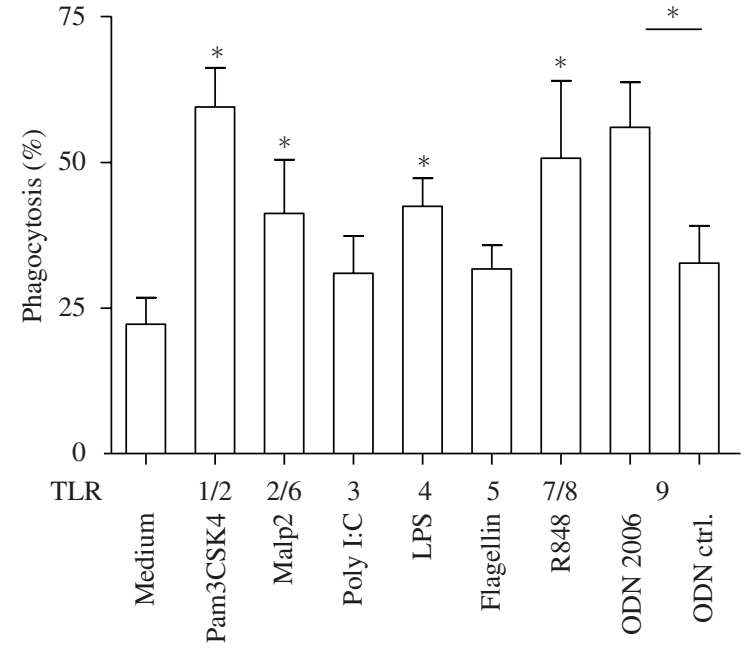

(a)

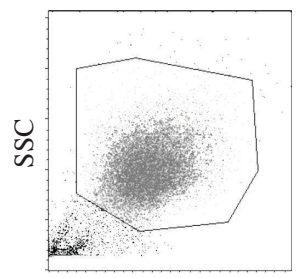

FSC

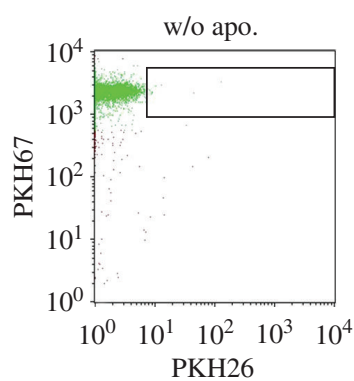

(d)

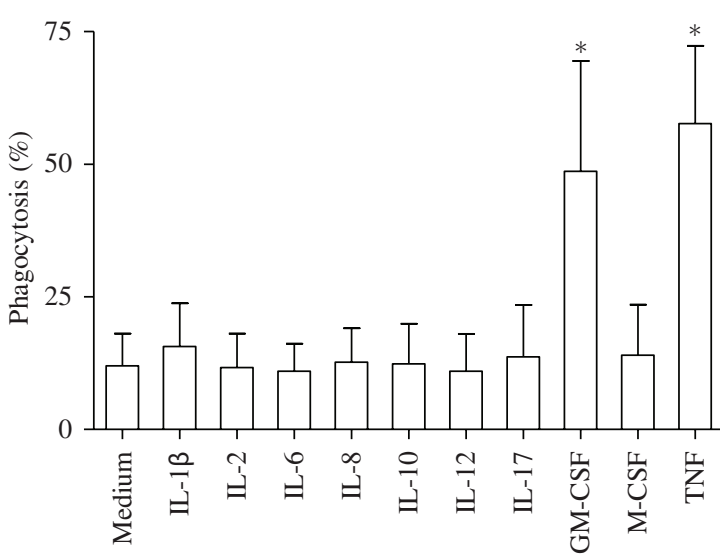

(b)

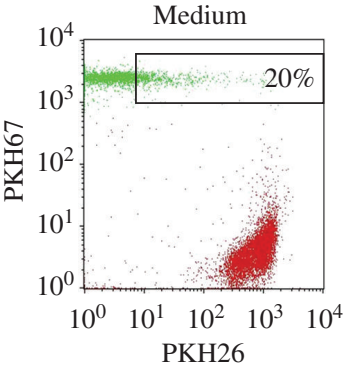

(e)

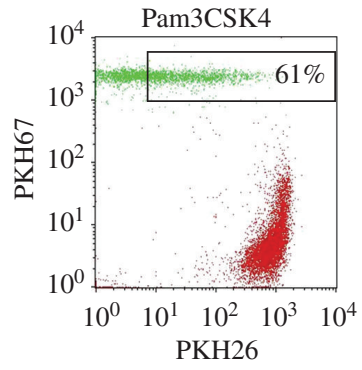

(f)

FIGURE 1: Phagocytosis of apoptotic neutrophils by purified human neutrophil granulocytes. $1 \times 10^{5}$ PKH67 stained viable neutrophils were preincubated with the indicated stimuli for 30 min and, subsequently, $4 \times 10^{5}$ PKH26 stained apoptotic neutrophils were added. Phagocytosis was assessed by flow cytometry after $90 \mathrm{~min}$. (a) Effect of TLR-ligands on the phagocytosis rate. (b) Effect of various cytokines on the phagocytosis rate. Data show mean \pm SD from 3 independent experiments, ${ }^{*}: P<0.05$. (c)(f) Representative dot-plots for the quantitative assessment of ingestion of apoptotic cells by purified neutrophils by using flow cytometry. (c) FSC/SSC gating of neutrophils. (d) Negative control: PKH67labeled (green) fresh neutrophils without apoptotic cells. (e) PKH67-labeled (green) fresh neutrophils after coincubation with PKH26-labeled (red) apoptotic neutrophils in medium alone. (f) Pam3CSK4-treated PKH67-labeled (green) fresh neutrophils after coincubation with PKH26-labeled (red) apoptotic neutrophils.

(Figure 1(a)). However, the TLR3 ligand poly I: C had no activating effect (Figure 1(a)). R848 and CpG DNA have previously shown to activate neutrophil functions $[8,9]$. In addition CpG DNA was reported to enhance the uptake of apoptotic cells also by macrophages [10]. The lack of activating effect of poly I:C can be explained with the fact that mature neutrophils do not express TLR3 [8].

\subsection{Exposure to GM-CSF and TNF Leads to Enhanced Phagocytosis of Apoptotic Cells by Neutrophils}

A large number of cytokines can be present in acutely infected/inflamed tissues. Freshly isolated neutrophils were exposed to various cytokines in order to investigate whether cytokines influence the capacity of neutrophils to ingest apoptotic cells. GM-CSF was used as positive control, since this proinflammatory 


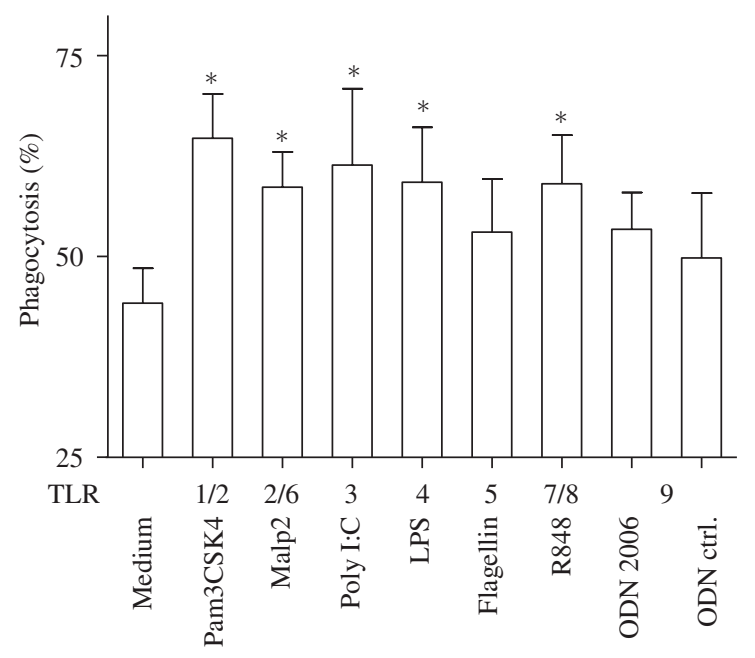

(a)

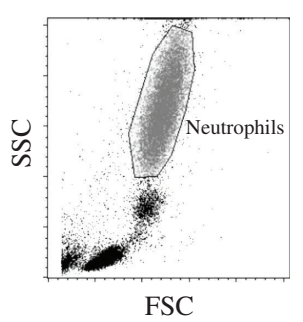

(c)

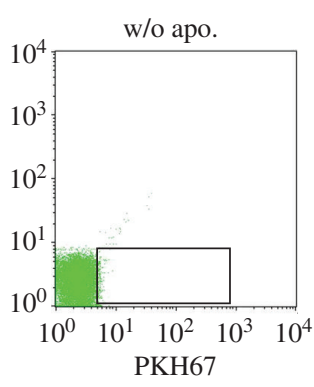

(d)

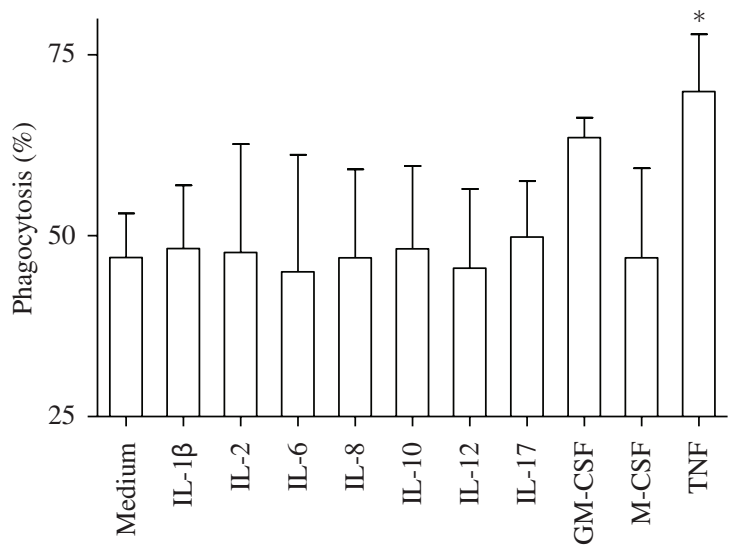

(b)

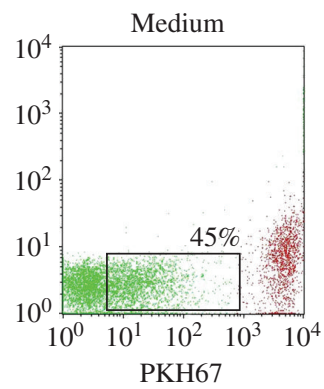

(e)

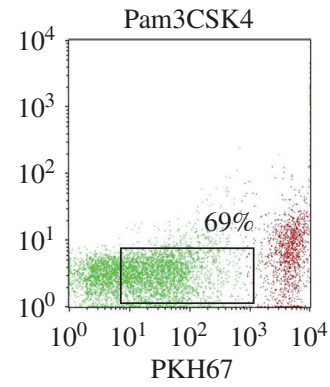

(f)

FIGURE 2: Phagocytosis of apoptotic neutrophils by neutrophils in whole blood. $50 \mu \mathrm{L}$ heparinized whole blood was preincubated with the indicated stimuli for $30 \mathrm{~min}$ and, subsequently, $1 \times 10^{6} \mathrm{PKH} 67$ stained apoptotic neutrophils were added. Phagocytosis was assessed after $60 \mathrm{~min}$ by using flow cytometry. (a) Effect of TLR-ligands on the phagocytosis rate. (b) Effect of various cytokines on the phagocytosis rate. Data show mean \pm SD from 3 independent experiments, ${ }^{*}: P<0.05$. (c)-(f) Representative dot-plots for the quantitative assessment of ingestion of apoptotic cells by neutrophils in whole blood by using flow cytometry. (c) FSC/SSC gating of neutrophils. (d) Negative control: gated neutrophils without apoptotic cells. (e) neutrophil phagocytosis of PKH67-labeled (green) apoptotic neutrophils. (f) Neutrophil phagocytosis of PKH67-labeled (green) apoptotic neutrophils after exposure to Pam3CSK4.

cytokine reportedly enhances the phagocytosis of apoptotic cells by neutrophils [5]. M-CSF was used as a negative control since mature neutrophils do not express the receptor for M-CSF.

Among the several cytokines tested solely GM-CSF and TNF enhanced the phagocytosis rate (Figure 1(b)). The cytokines IL-1 $\beta$, IL-2, IL-6, IL-8, IL-10, IL-12, and IL-17 had no effect on the ingestion of apoptotic cells by human neutrophils (Figure 1(b)).

GM-CSF and TNF are proinflammatory cytokines with known activating effects on neutrophil functions [5, 11]. IL-17 was shown not to influence neutrophil effector mechanisms [12], while other cytokines tested, for example, IL-1 $\beta$, IL-2, and IL-6 [13], can influence certain neutrophil functions. In our experiments, however, at least when applied alone, and not in combination with other activating/priming stimuli, these cytokines did not affect the capacity of neutrophils to phagocytose apoptotic cells. We cannot exclude the possibility that combination of more than one cytokine is required to achieve the modulation of 
apoptotic cell phagocytosis by neutrophils, since IL-2, for example, was reported to activate gene expression in neutrophils only in combination with GM-CSF [14].

\subsection{Neutrophil-Mediated Phagocytosis of Apoptotic Cells in Whole Blood}

Although neutrophils are recruited in large numbers into the inflamed/infected tissue, they are not the only leukocyte population present. Interactions between the various leukocytes may have an impact on neutrophil functions including their capacity to phagocytose apoptotic cells. Since we cannot simulate properly an inflamed tissue in vitro, experiments in whole blood were carried out to investigate a potential role of cell-cell interactions. This approach has certainly its limitations, but it allows interactions between various leukocyte populations.

Small volumes $(50 \mu \mathrm{L})$ of heparinized whole blood were treated with various TLR-ligands and cytokines for $30 \mathrm{~min}$ at $37^{\circ} \mathrm{C}$. Subsequently, fluorescently labeled apoptotic neutrophils were added to the blood samples. The erythrocytes were lysed, and the phagocytosis of apoptotic cells by neutrophils was assessed by flow cytometry after $60 \mathrm{~min}$.

The rate of phagocytosis was higher in whole blood than in the experiments using isolated neutrophils. More than $40 \%$ of the neutrophils in the whole blood assay ingested apoptotic material without stimulation (Figure 2). Similar to isolated neutrophils, the phagocytic rate of neutrophils in whole blood was significantly increased after exposure to Pam3CSK4, Malp-2, LPS, and R848 (Figure 2(a)). However, in contrast to isolated neutrophils, the effect of CpG-DNA (ODN2006) was not significant.

Importantly, though neutrophils do not express TLR3 [8], poly I: C strongly enhanced the phagocytosis rate of neutrophils in whole blood (Figure 2(a)) in contrast to the experiments with isolated neutrophils, which did not show a response to Poly I: C (Figure 1(a)). It is quite conceivable, that in the whole blood assay, poly I: C exerted an effect on other leukocyte populations and the interaction of neutrophils with these poly I: C-activated cells and/or with their secreted products led to the enhanced phagocytosis rate. Indeed poly I : $\mathrm{C}$ has been shown to activate monocyte and lymphocyte functions $[15,16]$. Monocytes have been identified as the major source of TNF in blood after poly I: C stimulation [15]. The observed activating effect of poly I: C in whole blood (Figure 2(a)) but not in experiments with purified neutrophils (Figure 1(a)) clearly indicates the importance of cell-cell interactions for the modulation of the ingestion of apoptotic cells by neutrophils.

The effects of cytokines in whole blood assays were similar to those observed in the experiments with isolated neutrophils (Figure 1(b)). Again the cytokines IL-1 $\beta$, IL-2, IL-6, IL-8, IL-10, IL-12, and IL17 did not enhance the phagocytosis rate of neutrophils (Figure 2(b)), whereas TNF significantly increased phagocytosis (Figure 2(b)). However, although GM-CSF showed a tendency to enhance phagocytosis, due to the high standard deviation in this set of experiments, the effect was not statistically significant.

At sites of acute infection/inflammation, neutrophils are exposed to microbial constituents and various cytokines. The results presented here strongly suggest that neutrophils activated by these proinflammatory stimuli acquire an enhanced phagocytic capacity and, consequently, can play a major role in the clearance of apoptotic neutrophils. Phagocytosis of apoptotic cells inhibits proinflammatory neutrophil functions [5]. Therefore, in addition to the physical removal of apoptotic cells, phagocytosis of apoptotic cells by neutrophils can contribute to the resolution of inflammation.

\section{ACKNOWLEDGMENTS}

This work was supported by DFG Excellence Cluster "Inflammation at Interfaces" (EXC 306/1) and by the Focus Program "Autoimmunity" at the University of Lübeck. The authors thank Ms. Sonja Möller for expert technical assistance and Dr. Inga Wilde for critical reading of the paper. This paper was presented in part at the 45th Annual Scientific Meeting of the European Society for Clinical Investigation, Crete, Greece, 13-16 April 2011. Lars Hellberg and Sabrina Fuchs contributed equally to this work. 


\section{REFERENCES}

[1] J. S. Savill, A. H. Wyllie, J. E. Henson, M. J. Walport, P. M. Henson, and C. Haslett, "Macrophage phagocytosis of aging neutrophils in inflammation. Programmed cell death in the neutrophil leads to its recognition by macrophages," The Journal of Clinical Investigation, vol. 83, no. 3, pp. 865-875, 1989.

[2] C. Haslett, J. S. Savill, M. K. Whyte, M. Stern, I. Dransfield, and L. C. Meagher, "Granulocyte apoptosis and the control of inflammation," Philosophical transactions of the Royal Society of London. Series B, vol. 345, no. 1313, pp. 327-333, 1994.

[3] L. P. Erwig and P. M. Henson, "Immunological consequences of apoptotic cell phagocytosis," American Journal of Pathology, vol. 171, no. 1, pp. 2-8, 2007.

[4] M. Scannell and P. Maderna, "Lipoxins and annexin-1: resolution of inflammation and regulation of phagocytosis of apoptotic cells," TheScientificWorldJOURNAL, vol. 6, pp. 1555-1573, 2006.

[5] L. Esmann, C. Idel, A. Sarkar et al., "Phagocytosis of apoptotic cells by neutrophil granulocytes: diminished proinflammatory neutrophil functions in the presence of apoptotic cells," Journal of Immunology, vol. 184, no. 1, pp. 391-400, 2010.

[6] G. Galati, P. Rovere, G. Citterio et al., "In vivo administration of GM-CSF promotes the clearance of apoptotic cells: effects on monocytes and polymorphonuclear leukocytes," Journal of Leukocyte Biology, vol. 67, no. 2, pp. 174-182, 2000.

[7] K. Rydell-Törmänen, L. Uller, and J. S. Erjefält, "Neutrophil cannibalism-a back up when the macrophage clearance system is insufficient," Respiratory Research, vol. 7, article 143, 2006.

[8] F. Hayashi, T. K. Means, and A. D. Luster, "Toll-like receptors stimulate human neutrophil function," Blood, vol. 102, no. 7, pp. 2660-2669, 2003.

[9] I. Wilde, S. Lotz, D. Engelmann et al., "Direct stimulatory effects of the TLR2/6 ligand bacterial lipopeptide MALP-2 on neutrophil granulocytes," Medical Microbiology and Immunology, vol. 196, no. 2, pp. 61-71, 2007.

[10] J. Wang, W. L. Huang, and R. Y. Liu, "CpG-ODN enhances ingestion of apoptotic neutrophils by macrophages," Clinical and Experimental Medicine, vol. 9, no. 1, pp. 37-43, 2009.

[11] M. M. Moxey-Mims, H. H. Simms, M. M. Frank, E. Y. Lin, and T. A. Gaither, "The effects of IL-1, IL-2, and tumor necrosis factor on polymorphonuclear leukocyte Fc $\gamma$ receptor-mediated phagocytosis: IL-2 down-regulates the effect of tumor necrosis factor," Journal of Immunology, vol. 147, no. 6, pp. 1823-1830, 1991.

[12] M. Pelletier, L. Maggi, A. Micheletti et al., "Evidence for a cross-talk between human neutrophils and Th17 cells," Blood, vol. 115, no. 2, pp. 335-343, 2010.

[13] D. V. Pechkovsky, M. P. Potapnev, and O. M. Zalutskaya, "Different patterns of cytokine regulation of phagocytosis and bacterial killing by human neutrophils," International Journal of Antimicrobial Agents, vol. 7, no. 1, pp. 33-40, 1996.

[14] D. Girard, J. Gosselin, D. Heitz, R. Paquin, and A. D. Beaulieu, "Effects of interleukin-2 on gene expression in human neutrophils," Blood, vol. 86, no. 3, pp. 1170-1176, 1995.

[15] M. Rosas-Ballina, R. S. Goldstein, M. Gallowitsch-Puerta et al., "The selective $\alpha 7$ agonist GTS-21 attenuates cytokine production in human whole blood and human monocytes activated by ligands for TLR2, TLR3, TLR4, TLR9, and RAGE," Molecular Medicine, vol. 15, no. 7-8, pp. 195-202, 2009.

[16] T. Reimer, M. Brcic, M. Schweizer, and T. W. Jungi, "poly(I:C) and LPS induce distinct IRF3 and NF- $\kappa$ B signaling during type-I IFN and TNF responses in human macrophages," Journal of Leukocyte Biology, vol. 83, no. 5, pp. 1249-1257, 2008.

\section{This article should be cited as follows:}

Lars Hellberg, Sabrina Fuchs, Christoph Gericke, Arup Sarkar, Martina Behnen, Werner Solbach, and Tamás Laskay, "Proinflammatory Stimuli Enhance Phagocytosis of Apoptotic Cells by Neutrophil Granulocytes," TheScientificWorldJOURNAL, vol. 11, pp. 2230-2236, 2011. 


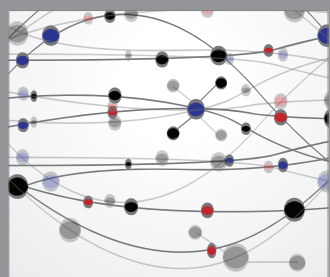

The Scientific World Journal
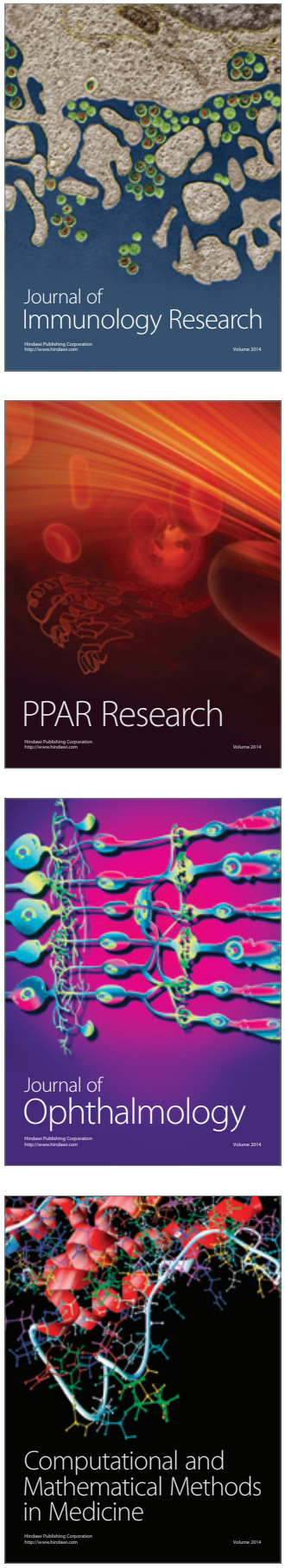

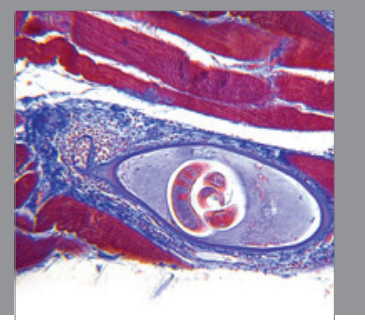

Gastroenterology

Research and Practice
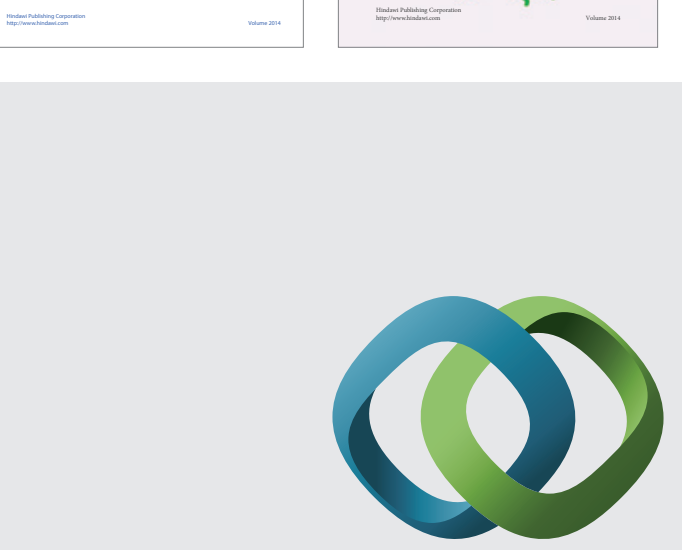

\section{Hindawi}

Submit your manuscripts at

http://www.hindawi.com
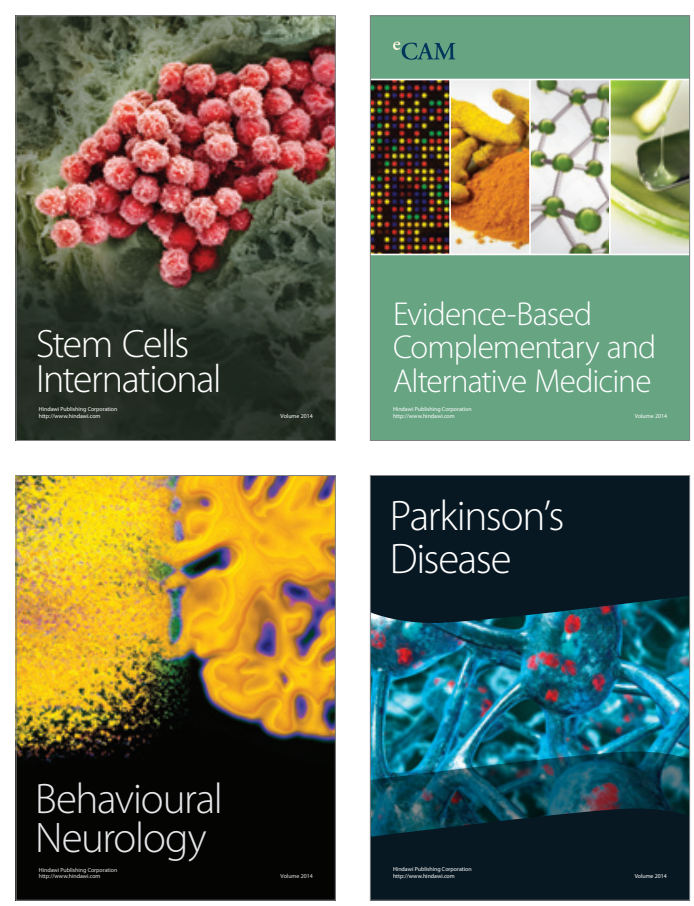

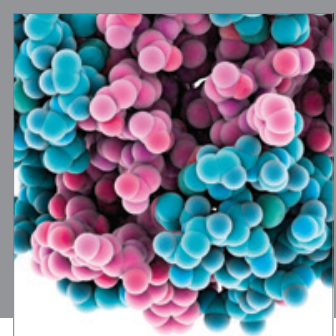

Journal of
Diabetes Research

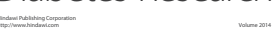

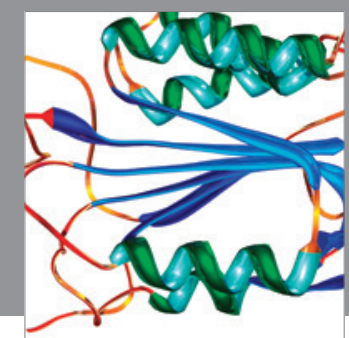

Disease Markers
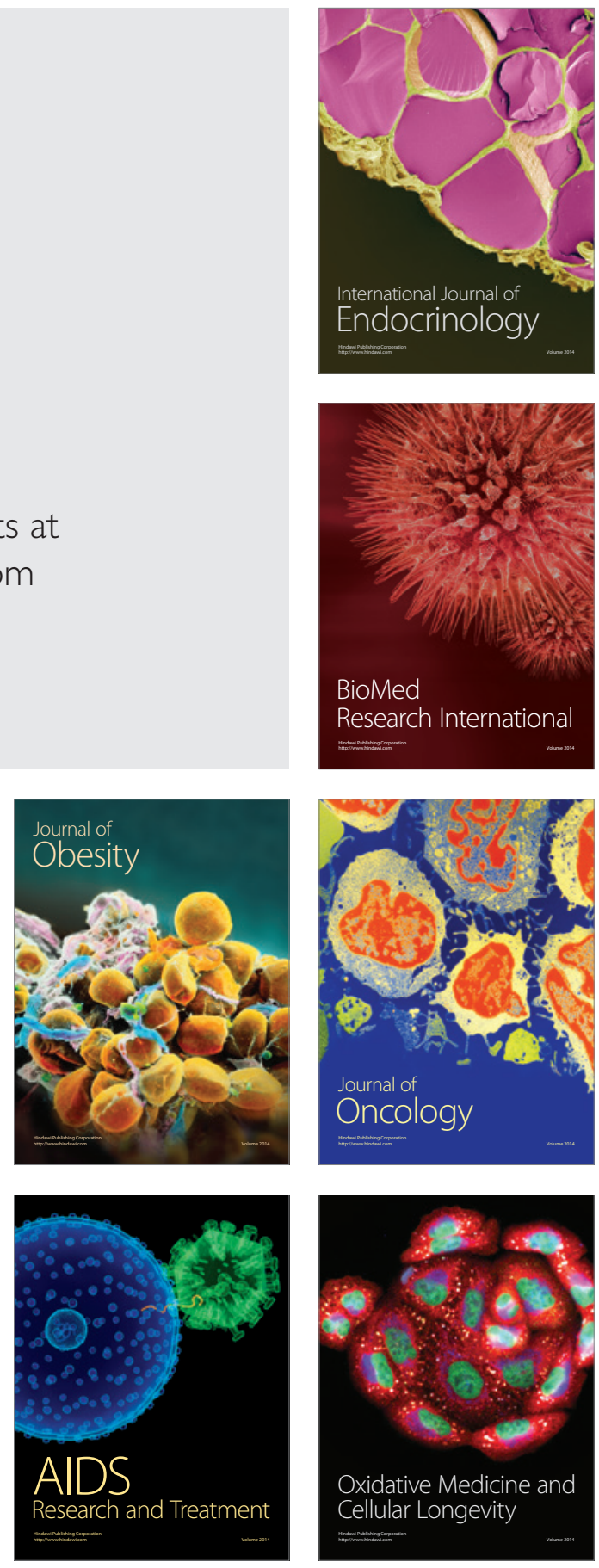